\title{
Refinement in Captcha as Graphical Password using Image Grid Techniques
}

\author{
Puja Mashalkar $\mathbf{A}^{\mathbf{1}}$, Mate Rutuja $\mathbf{N}^{2}$, Pawar Vaishali $\mathbf{B}^{3}$, Setia Pooja $\mathbf{R}^{4}$, Minal Shahakar \\ Pad. Dr D.Y Patil Institute of Engineering \& Technology, Pimpri, Pune, India \\ Savitribai Phule University
}

\begin{abstract}
Generally security problems deals with the hard AI problems related to security as emerging paradigm which has been unexplored. Captcha is a technology which presents security primitives for mathematical problems. Carp addresses the security problems which are based on the online attacks, attacks made as relay and surfing attacks. In CaRP password is only searched by best online guessing attacks which can be available in password sets. Captcha technology, which we can describe as Captcha which can be represented as graphical passwords. CaRP is defined as both Captcha and a password as a graphical scheme. CaRP also delivers the idea such as Pass Point, which often describes the weak passwords. CaRP is not a method but it offers reasonable security and usability thus appears to fit well with some practical applications for improving the problems related to online security.
\end{abstract}

Keywords: Graphical Passwords, Hotspot, Captcha, Security Primitives.

\section{INTRODUCTION}

Captcha is the most notable primitive invented, which Psychological Authentication [2] requires a client to distinguishes human users from computers by presenting a produce a way through a board of pictures as takes after: challenge, i.e., a puzzle, beyond the capability of beginning from the upper left picture, moving down if the computers but easy for humans. Captcha is now a standard picture is in portfolio, or right generally an Internet security technique to protect online email and acknowledgment based plan requires distinguishing baits other services from being abused by bots and this of the visual articles fitting in with a watchword portfolio. paradigm has achieved just a limited success as compared A commonplace plan is Pass faces [2] wherein a client with the cryptographic primitives based on hard math chooses a arrangement of countenances from a database in problems and their wide applications. When one making a secret key. A mid verification, a board of CAPTCHA scheme is broken, a new and more secure one hopeful appearances is displayed for the client to choose may appear and be converted to a CaRP scheme. Due to the face fitting in with her portfolio. This procedure is reasonable security and usability and practical rehashed a few adjusts, every round with an alternate applications, CaRP has good potential for refinements.

Countless secret key plans have been proposed. They can be grouped into three classifications concurring to the errand included in retaining and entering passwords: acknowledgment, review, and signaled review. Every sort will be quickly depicted here. More can be found in a late audit of graphical pass- words. An acknowledgment based plan requires distinguishing among baits the visual articles fitting in with a watchword portfolio. A commonplace plan is Pass faces [2] wherein a client chooses a arrangement of countenances from a database in making a secret key. A mid verification, a board of hopeful appearances is displayed for the client to choose the face fitting in with portfolio. This procedure is rehashed a few adjusts, every round with an alternate board. A fruitful login requires right determination in each round.

The arrangement of pictures in a board continues as before between logins, however their ar- eas are permuted. Story [2] is comparative to Pass faces however the pictures in the portfolio are requested, and a client must distinguish her portfolio pictures in the right request. A sensation that this has happened before [2] is likewise comparative however utilizes an extensive arrangement of computer generated. "irregular craftsmanship" pictures. board. A fruitful login requires right determination in each round.

Security primitives are based on hard mathematical problems. Using hard AI problems for security is emerging as an exciting new paradigm, but has been underexplored. A fundamental task in security is to create cryptographic primitives based on hard mathematical problems that are computationally intractable. This paper shows a new security primitive based on hard AI problems, namely, a novel family of graphical password systems built on top of Captcha technology that is called Captcha as graphical passwords (CaRP). CaRP is both a Captcha and a graphical password scheme. CaRP addresses a number of security problems altogether, such as online guessing attacks, relay attacks, and, if combined with dual-view technologies, shoulder-surfing attacks. Notably, a CaRP password can be found only probabilistically by automatic online guessing attacks even if the password is in the search set.

CaRP also offers a novel approach to address the wellknown image hotspot problem in popular graphical password systems, such as Pass Points, that often leads to weak password choices. CaRP is not a panacea, but it offers reasonable security and usability and appears to fit 
well with some practical applications for improving online are autonomous of center of-consideration models, and security. An exemplary CaRPs built on both text Captcha depend on picture autonomous speculating examples. and image-recognition Captcha. One of them is a text CaRP wherein a password is a sequence of characters like a text password, but entered by clicking the right character sequence on CaRP images. CaRP offers protection against online dictionary attacks on passwords, which have been for long time a major security threat for various online services. This threat is widespread and considered as a top cyber security risk. Defense against online dictionary attacks is a more subtle problem than it might appear.

\section{RELATED WORK}

R. Biddle, S. Chiasson, and P. C. van Oorschot [1] indexes the existing methodologies, highlights the novel components of chose plans and distinguishing key use for security preferences. Mechanized Turing Tests (ATTs), otherwise called human-tuned in strategies, and were as of late utilized in a login convention by Pinkas and Sander (2002) to secure against online secret key speculating assaults. System display modifications giving another history-based login convention with ATTs, which uses fizzled login tallies.

P.C. Van Oorschot and S. Stubblebine [3] present modifications providing a new history-based login protocol with ATTs, which uses failed-login counts. Analysis indicates that the new protocol offers opportunities for improved security and user-friendliness.

I. Jermyn, A. Mayer, F. Monrose, M. Reiter, and A. Rubin [4] propose a new approach for authentication that improvises the notion of text password to Graphical password. Designed with the same purpose of providing security, graphical password differs with textual password in the way by adding image or handwritten text to the original text password thus implementing more security.

"Pass-Go: A proposal to improve the usability of Graphical passwords," by H. Tao and C. Adams [5] proposes the method as roused by an old Chinese amusement as a pass go in which a particular user chooses the main crossing points which can be based on the matrix which a novel approach to input as a secret word.

This system, assess different systems for simply robotized assaults against Pass Points style graphical passwords. For producing these assaults, a diagram based calculation is used with efficiently make word references in light of heuristics. Some systems consolidate snap request heuristics with center of-consideration sweep ways created from a computational model of visual consideration, yielding significantly preferable mechanized assaults over past work.

Computerized assaulted 7-16 percent of passwords for two delegate pictures utilizing word references of roughly where the full secret key space is 2 . Unwinding snap request designs considerably expanded the assault efficacy though with bigger word references of around, permitting assaults that speculated 48-54 percent of passwords contrasted with past aftereffects of 1 percent and 9 percent on the same dataset for two pictures. These last assaults

\section{SYSTEM DESIGN}

A security primitive in view of hard AI issues, to be specific, is a novel group of graphical secret key frameworks based on top of Captcha innovation, which we call Captcha as graphical passwords (CaRP).

CaRP is both a Captcha and a graphical secret key plan. $\mathrm{CaRP}$ addresses various security issues by and large, for example, web speculating assaults, hand-off assaults, and, if joined with double view innovations, shoulder-surfing assaults. Prominently, a CaRP secret key can be discovered just probabilistically via programmed web speculating assaults regardless of the fact that the watchword is in the inquiry set.

CaRP likewise offers a novel way to deal with location the understood picture hotspot issue in prevalent graphical secret word frameworks, for example, PassPoints, that frequently prompts frail watchword decisions. CaRP is not a panacea, but rather it offers sensible security and ease of use and seems to fit well with some down to earth applications for enhancing online security.

This paper shows an excellent CaRPs based on both content Captcha and picture acknowledgment Captcha. One of them is a content CaRP wherein a watchword is an arrangement of characters like a content secret key, however entered by tapping the right character grouping on CaRP pictures. CaRP offers assurance against online lexicon assaults on passwords, which have been for long time a noteworthy security danger for different online administrations.

This danger is far reaching and considered as a top digital security hazard. Safeguard against online word reference assaults is a more inconspicuous issue than it may show up.

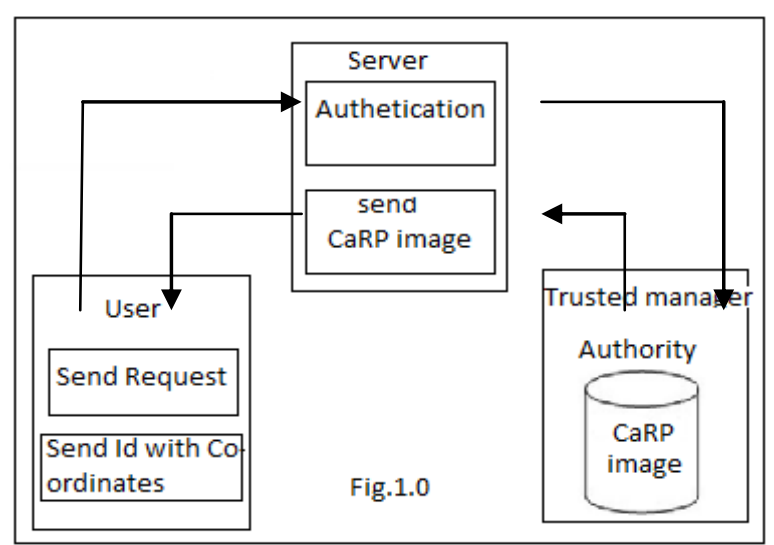

Fig1. Architectural Design

Fig. 1 describes the flow which relates the file transferring through unique methods as encryptions and decryption using a smart object. Wherein, the particular user selects the unique object to be recognized further by the next user while receiving the file which is suitably shared through the methods of unique encrypted format. 


\section{TECHNIQUES OF CAPTCHA}

\section{Graphical Password}

In this paper, users are having authentication and security to access the detail which is presented in the Image system. Before accessing or searching the details user should have the account in that otherwise they should register first.

\section{CAPTCHA in Authentication}

To use both Captcha and password in a user authentication protocol is called as Captcha-based Password Authentication (CbPA) protocol, to counter online dictionary attacks. The CbPA-protocol in requires solving a Captcha challenge after inputting a valid pair of user ID and password unless a valid browser cookie is received. For an invalid pair of user ID and password, the user has a certain probability to solve a Captcha challenge before being denied access.

\section{Thwart Guessing Attacks}

In guessing attack, a password guess tested in an unsuccessful trial is determined wrong and excluded from subsequent trials. The number of undetermined password guesses decreases with more trials, leading to a better chance of finding the password.

To counter guessing attacks, traditional approaches in designing graphical passwords aim at increasing the effective password space to make passwords harder to guess and thus require more trials. No matter how secure a graphical password scheme is, the password can always be found by a brute force attack.

In this paper, two types are distinguished of guessing attacks: automatic guessing attacks apply an automatic trial and error process but S can be manually constructed whereas human guessing attacks apply a manual trial and error process.

\section{Security of Underlying Captcha}

Computational intractability in recognizing objects in $\mathrm{CaRP}$ images is fundamental to CaRP. Existing analyses on Captcha security were mostly case by case or used an approximate process. No theoretic security model has been established yet. Object segmentation is considered as a computationally expensive, combinatorial-hard problem, which modern text Captcha schemes rely on.

\section{CONCLUSION}

$\mathrm{CaRP}$ is another security primitive depending on unsolved hard AI issues. CaRP is both a Captcha and a graphical secret word plan. The thought of CaRP presents another group of graphical passwords, which receives another way to deal with counter web speculating assaults: another CaRP picture, which is additionally a Captcha test, is utilized for each login endeavor to make trials of an internet speculating assault computationally free of one another. A secret key of CaRP can be discovered just probabilistically via programmed web speculating assaults including animal power assaults, a fancied security property that other graphical secret key plans need. Hotspots in CaRP pictures can never again be abused to mount programmed web speculating assaults, an inalienable powerlessness in numerous graphical secret key frameworks. CaRP powers foes to depend on fundamentally less proficient and considerably more exorbitant human-based assaults. Notwithstanding offering insurance from internet speculating assaults, CaRP is additionally impervious to Captcha hand-off assaults, and, if consolidated with double view advancements, shouldersurfing assaults. CaRP can likewise decrease spam messages sent from a Web email administration.

\section{REFERENCES}

[1] R. Biddle, S. Chiasson, and P. C. van Oorschot, "Graphical passwords: Learning from the first twelve years," ACM Comput. Surveys, vol. 44, no. 4, 2012.

[2] (2012, Feb.). The Science Behind Pass faces [Online].Available:http//www.realuser.com/published/Science BehindPasfaces.pdf

[3] P. C. van Oorschot and S. Stubblebine, "On countering online dictionary attacks with login histories and humans-in-the-loop," ACM Trans. Inf. Syst. Security, vol. 9, no. 3, pp. 235-258, 2006

[4] I. Jermyn, A. Mayer, F. Monrose, M. Reiter, and A. Rubin," The design and analysis of Graphical Password" in Proc. $8^{\text {th }}$ USENIX in security symp. 1999, pp. 1-15.

[5] H. Tao and C. Adams "Pass-Go: A proposal to improve the usability of graphical passwords," Int. J. Netw. Security, vol. 7, no. 2, pp. 273-292, 2008.

[6] P. C. van Oorschot and J. Thorpe, "On predictive models and user drawn graphical passwords," ACM Trans. Inf. Syst. Security, vol. 10 , no. 4 , pp. $1-33,2008$.

[7] J. Thorpe and P. C. van Oorschot, "Human-seeded attacks and exploiting hot spots in graphical passwords," in Proc. USENIX Security, 2007, pp. 103-118.

[8] A. E. Dirik, N. Memon, and J.-C. Birget, "Modeling user choice in the pass point's graphical password scheme," in Proc. Symp. Usable Privacy Security, 2007, pp. 20-28.

[9] P. C. van Oorschot and J. Thorpe, On predictive models and userdrawn graphical passwords, ACM Trans. Inf. Syst. Security, vol. 10 , no. 4, pp. 133, 2008.

[10] A. E. Dirik, N. Memon, and J.-C. Birget, Modeling user choice in the passpoints graphical password scheme, in Proc. Symp. Usable Privacy Security, 2007, pp.2028.

[11] D. M. Blei, A. Y. Ng, and M. I. Jordan, Latent dirichlet allocation, the Journal of machine Learning research, vol. 3, pp. 9931022, 2003.

[12] P. C. van Oorschot and J. Thorpe, Exploiting predictability in click based graphical passwords, J. Comput. Security, vol. 19, no. 4, pp. 669702,2011

[13] T.Wolverton. (2002, Mar. 26). Hackers Attack eBay Accounts [Online]. Available: http://www.zdnet.co.uk/news/networking/2002/03/26/hackersattack-ebay-accounts-2107350/ 